\title{
Tip- and Laser-based 3D Nanofabrication in Extended Macroscopic Working Areas
}

\author{
Ingo Ortlepp ${ }^{1}$ (D) Thomas Fröhlich ${ }^{1} \cdot$ Roland FüßI $^{1} \cdot$ Johann Reger $^{2} \cdot$ Christoph Schäffel $^{11}$ - Stefan Sinzinger ${ }^{3}$. \\ Steffen Strehle ${ }^{4} \cdot$ René Theska $^{5}$ - Lena Zentner ${ }^{6}$ Jens-Peter Zöllner ${ }^{7} \cdot$ Ivo W. Rangelow $^{8} \cdot$ Carsten Reinhardt $^{12}$. \\ Tino Hausotte ${ }^{13} \cdot$ Xinrui Cao $^{3}$. Oliver Dannberg ${ }^{1} \cdot$ Florian Fern $^{14}$. David Fischer ${ }^{3}$. Stephan Gorges ${ }^{11}$. \\ Martin Hofmann ${ }^{4}$. Johannes Kirchner ${ }^{15} \cdot$ Andreas Meister $^{9} \cdot$ Taras Sasiuk $^{1} \cdot$ Ralf Schienbein $^{5} \cdot$ Shraddha Supreeti $^{10}$. \\ Laura Mohr-Weidenfeller ${ }^{4} \cdot$ Christoph Weise $^{2} \cdot$ Christoph Reuter $^{4} \cdot$ Jaqueline Stauffenberg $^{1} \cdot$ Eberhard Manske $^{1}$
}

Received: 8 March 2021 / Revised: 22 June 2021 / Accepted: 23 June 2021 / Published online: 10 August 2021

(c) The Author(s) 2021

\begin{abstract}
The field of optical lithography is subject to intense research and has gained enormous improvement. However, the effort necessary for creating structures at the size of $20 \mathrm{~nm}$ and below is considerable using conventional technologies. This effort and the resulting financial requirements can only be tackled by few global companies and thus a paradigm change for the semiconductor industry is conceivable: custom design and solutions for specific applications will dominate future development (Fritze in: Panning EM, Liddle JA (eds) Novel patterning technologies. International society for optics and photonics. SPIE, Bellingham, 2021. https://doi.org/10.1117/12.2593229). For this reason, new aspects arise for future lithography, which is why enormous effort has been directed to the development of alternative fabrication technologies. Yet, the technologies emerging from this process, which are promising for coping with the current resolution and accuracy challenges, are only demonstrated as a proof-of-concept on a lab scale of several square micrometers. Such scale is not adequate for the requirements of modern lithography; therefore, there is the need for new and alternative cross-scale solutions to further advance the possibilities of unconventional nanotechnologies. Similar challenges arise because of the technical progress in various other fields, realizing new and unique functionalities based on nanoscale effects, e.g., in nanophotonics, quantum computing, energy harvesting, and life sciences. Experimental platforms for basic research in the field of scale-spanning nanomeasuring and nanofabrication are necessary for these tasks, which are available at the Technische Universität Ilmenau in the form of nanopositioning and nanomeasuring (NPM) machines. With this equipment, the limits of technical structurability are explored for high-performance tip-based and laser-based processes for enabling real 3D nanofabrication with the highest precision in an adequate working range of several thousand cubic millimeters.
\end{abstract}

Keywords Nanomeasuring $\cdot$ Nanopositioning $\cdot$ Nanomanufacturing $\cdot$ Scale-spanning $\cdot$ Tip-based $\cdot$ Laser-based . Nanofabrication

\section{Introduction}

The semiconductor industry has been following Moore's law [2] with surprising steadiness for 55 years. A fundamental challenge is that the fabrication technologies must increasingly be able to routinely measure, control, and structure at the atomic scale. If the further development of optical lithography (EUV) [3] continues with the rate of the past

Ingo Ortlepp

ingo.ortlepp@tu-ilmenau.de

Extended author information available on the last page of the article decades, not only the technical challenges but also the costs will increase almost immeasurably [4]. Therefore, it is no question whether, but how the continuous reduction in size can be realized in the coming years.

Despite the massive development of optical lithography, it is evident that structure sizes of $<20 \mathrm{~nm}$ can only be achieved with great effort using known methods [5, 6]. Here, tip-based nanofabrication processes, which already enable structuring in the sub-10-nm range, offer great potential. However, these processes have only been demonstrated in small processing areas (a few $100 \mu \mathrm{m}^{2}$ ), at low speeds and with limited precision [7]. Certain optical processes allow 
for sub-wavelength structuring $[8,9]$, though only in the sense of functionalized, plane surfaces.

Similarly, nanotechnologies are advancing in many other fields to solve essential issues of health [10], agriculture [11], energy and resources [12], as well as environmental and climate protection [13]. However, for the implementation of many nanotechnological fabrication techniques, there is a lack of adequate measuring and positioning techniques that span scales of subnanometers to several centimeters.

In recent years, nanopositioning and nanometrology have experienced an enormous progression at the Technische Universität Ilmenau, especially regarding the further development of 3D precision measurement technology [14].

Consequently, within a framework of a DFG research training group on Tip-and laser-based 3D nanofabrication in extended macroscopic working areas, research is being conducted to combine nanofabrication processes with the outstanding capabilities of nanopositioning and nanomeasurement machines of the Technische Universität Ilmenau in such a way that new, alternative, and cross-scale solutions advance the possibilities of nanotechnologies. There, the limits of technical structurability are explored for high-performance tip-based processes such as atomic force microscopy (AFM). Furthermore, laser-based sub-wavelength methods, in conjunction with NPM technology, should enable real 3D nanofabrication of the highest precision. In this context, the possibilities of high-level parallelization of nanotools are of great interest from a manufacturing perspective. Nanostructuring on curved precision surfaces is also a major challenge and can lead to completely new technical solutions, e.g., the combination of photonic structures with precision optics.

\section{Motivation}

Nanofabrication aims for the production of nanoscale structures that achieve new functionalities as components or systems with defined (nanoscale) properties. According to the International Roadmap for Devices and Systems (IRDS) [6], the rate of development in the semiconductor industry is unbroken, and the resulting challenges are enormous. The greatest difficulties become apparent for upcoming sub10-nm technologies, where EUV lithography will certainly face serious technological issues and approach its fundamental size limits. Concurrently, there is enormous cost pressure for high-performance patterning techniques, which intensifies the search for economically viable solutions.

In addition to the advance of manufacturing technologies into the atomic range, it is increasingly important to enable the flexible and cost-effective production of components with complex geometries and a cross-scale mix of macroto micro- to nano-scale structures, even in smaller quantities.
Therefore, international research is being conducted in new, unconventional approaches, such as tip-based nanofabrication [15], new highly efficient e-beam processes [16] or nanoimprinting [17]. These technologies are currently advancing into molecular and atomic ranges with increasing complexity and 3D requirements. New solutions to continue the development in the semiconductor industry are only one aspect of nanofabrication. Advances should also ease and support the development of new fields such as nanoplasmonics [18], nanophotonics [19], nanobiology [20], nanomedicine [21], and others. This includes techniques for "BeyondCMOS devices", the realization of novel functionalities and unique properties based on nanoscale effects, the increase of optical resolution [22], the increase of the potential for energy harvesting or the revolution in computer and consumer electronics of quantum computing and nanophotonics. Furthermore, new solutions for the life sciences, from overcoming the nanoscale nature of DNA, viruses and protein molecules to nanomedicine for early detection, diagnosis and monitoring and therapy of diseases (protein and peptide delivery, nanorobots and nanoprobes, antibody therapeutics and even cell repair) have to be addressed [23, 24].

In recent years, a broad range of approaches to tip-based nanofabrication and laser-based sub-wavelength fabrication have been developed and investigated under this objective. The feasibility of sub-10-nm structuring [23] up to atomically precise "top-down" fabrication [25] could be demonstrated as "proof of concept" based on scanning probe techniques, however, most are only in areas up to $100 \mu \mathrm{m} \times 100 \mu \mathrm{m}$ [26]. The application of such techniques in large areas has not yet been demonstrated and poses a variety of challenges. The need for nanopositioning technology with exceptional performance for tip-based nanofabrication has been recognized without offering any applicable solutions so far [24]. There seems to be a stagnation in the Roadmap for Tip-based Nanofabrication postulated by TsENG [15, 24].

Technical implementation of such new structuring technologies in large areas has so far obviously failed because of a lack of adequate measurement and positioning technology that spans from subnanometers to several centimeters. The difficulties and challenges with regard to precision, reproducibility, dynamics, and stability are well known [7, 24]. Still, there is a great need for research into the flexible, cross-scale, and at the same time cost-effective production of complex nanoscale structures, even in smaller quantities.

The increasing demand for functionalized surfaces planar as well as non-planar - also entails the necessity to apply alternative processes for nanostructuring to modify the geometric, optical, mechanical, electrical, or magnetic material and surface properties. In this context, microstructuring and nanostructuring on curved technical surfaces poses a particularly great challenge. The functional combination of nanostructures with precision optics (aspheres, 
free-form surfaces) offers enormous potential for new hightech applications. The specific challenge of functionalized surfaces is highly linked to the combination of the latest micro/nano-machining technologies with highly developed nano-positioning and nano-measurement technology.

Considering the research deficits outlined, an essential task is to combine the latest micro- and nanofabrication processes with the enormous advantages of nanopositioning and nanometrology. For that purpose, nanopositioning and nanomeasuring machines (NPM machines), developed at the Technische Universität Ilmenau, are available, which have been proving their capability for nanomeasuring tasks since several years [27]. Based on the experiences regarding the position stability and trajectory fidelity of these machines, the attempt to use them for nanomanufacturing is obvious. When combining the advantages of advanced tip- and laserbased processes with the extraordinary positioning capabilities of NPM machines, there is the potential to push the limits of patternability, with sub-nanometer performance, across scales up to areas of $200 \mathrm{~mm} \times 200 \mathrm{~mm}$. Based on this synergy, true 3D nanofabrication (e.g. two-photon polymerization) and nanostructuring of 3D surfaces (precision optics, aspheres, and free-form surfaces) will become possible. This results in two fundamental goals:

1. investigation and further development of existing or the development of new micro-/nanofabrication processes for application in NPM machines, making use of their unique advantages, especially with regard to nanometer precision in large spatial ranges.

2. investigation and further development of nanopositioning and nanometrology with regard to new approaches to real 3D nanofabrication.

Research on these goals and challenges is currently carried out in the scope of the research training group (RTG) "Tip- and laser-based 3D nanofabrication in extended macroscopic working areas NanoFab", based on DFG funding. This consolidation of ten departments, leading in their field at the Technische Universität Ilmenau, aims to overcome the current and future barriers for promising alternative nanofabrication technologies at the wafer scale.

\section{State of the Art}

The demonstration of new tip-based fabrication techniques is often based on (standard) AFM, thus in small areas of $2 \mu \mathrm{m} \times 2 \mu \mathrm{m} \ldots 100 \mu \mathrm{m} \times 100 \mu \mathrm{m}$ [28], often with inadequate metrology or control technology. However, the problems of these AFM technologies are well known and beyond question:
"The size accuracy or dimension variation is critical since the ability to fabricate a specific structure with a specific dimension dictates the use of the unique dimension-dependent properties in nano-devices. The size control capability is absent in many existing TBN methods." [29].

Existing laser-based methods are also limited by the use of piezo scanners (extended by stepper motors) or mirror scanners. A transfer to large machining areas with high lateral structural accuracy and high positioning dynamics is not trivial and represents a serious research deficit.

To investigate tip-based nanofabrication technologies at the limits of structurability $(1-5 \mathrm{~nm})$ and to demonstrate them by using selected technologies, in particular singlenanometer lithography [30], it is necessary to achieve the highest manufacturing precision with ever-smaller structures in large areas with high dynamics. For that purpose, positioning systems which fulfil the requirement of placing the measuring or manufacturing tool with nanometer uncertainty in a working volume in the centimeter range are necessary. The nanopositioning and nanomeasuring machines, developed at the Technische Universität Ilmenau through many years of research in the field of nanometrology, can be used to address this task.

One of the first NPM machines capable of a nanopositioning in a large working volume was the NMM-1 [31]. This machine offers a positioning volume of $25 \mathrm{~mm} \times 25 \mathrm{~mm} \times 5 \mathrm{~mm}$ with a resolution of $0.1 \mathrm{~nm}$ and an axis positioning uncertainty of $3 \mathrm{~nm}$ [32]. The machine is based on the sample scanning mode, where the probe or tool as well as the measuring system are fixed to a metrological frame while the sample is moved in three spatial directions. The position of the sample is tracked by three fiber-coupled laser interferometers with a laser wavelength stability of $<2 \cdot 10^{-9}$, whose measuring axes virtually intersect at the center point of the tool/probe (tool center point, TCP) at any time. For this purpose, a special arrangement of the driving, guiding and measurement systems was designed. With this special setup, the Abbe principle is fulfilled in all three measuring directions, over the entire range of motion, at any time [32]. This results in the effective prevention of first-order measuring errors, which, among other measures, originates the high precision of the NMM-1 over the entire positioning range.

The basic functional design of the NMM-1 is shown in Fig. 1, with the moving measuring object carried by a corner mirror that is traced by three laser interferometers.

The implementation of the 3D Abbe principle and the prevention of first-order measuring errors is the basis for the high measuring capabilities of the NMM- 1 . To further enhance the measuring uncertainty, the tilt angles of the machine table are additionally closed-loop controlled [33]. This is realized by two autocollimators, detecting the rotation of the table around the $x$ - and $y$-axis (Nos. 4 and 5 in 

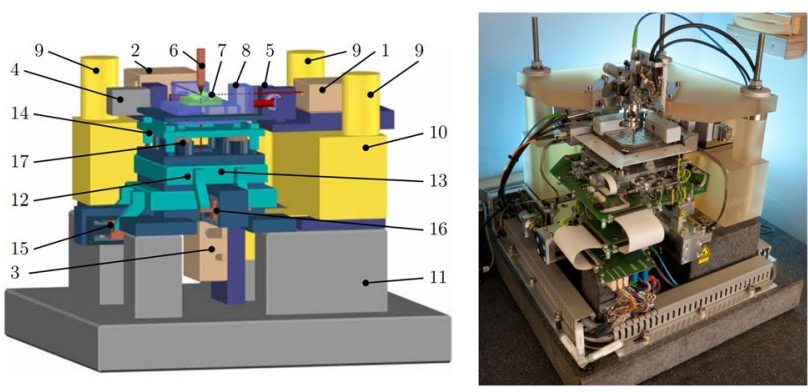

Fig. 1 Basic mechanical and optical design of the NMM-1. $1 x$-interferometer; $2 y$-interferometer; $3 z$-interferometer; 4 roll and yaw angle sensor; 5 pitch and yaw angle sensor; 6 probe; 7 sample; 8 corner mirror; 9 zerodur pillar; 10 metrology frame (Zerodur@); 11 base, guides of the $12 x$-axis, $13 y$-axis, $14 z$-axis and drives of the 15 $x$-axis, $16 y$-axis, $17 z$-axis $[34,37]$

Fig. 1) and the arrangement of the four $z$-driving systems, allowing active tilt control. This way, the machine table can be moved exactly parallel in the complete working volume of the NMM-1. Thus, the inherently limited accuracy of the mechanical guiding systems for the $x$-, $y$-, and $z$-movement, does not affect the overall machine precision, as the inevitable deviations are compensated for by the closed-loop machine control system. The prevented tilt of the machine table reduces measuring deviations even further by eliminating second-order measuring errors [34].

Based on the excellent measuring results and the experience gained with the challenges in nanometrology, an advanced NPM machine with an extended positioning range of $200 \mathrm{~mm} \times 200 \mathrm{~mm} \times 25 \mathrm{~mm}$, the NPMM-200 [35] (Fig. 2), was developed at the Technische Universität Ilmenau. This machine features the same basic concept as the NMM-1 and offers the same advantages regarding positioning accuracy and measuring capabilities. Because of the large operating volume, the closed-loop tilt angle control of the machine table is an essential issue. Therefore, the measuring of the $\varphi_{x}$ and $\varphi_{y}$ angles is realized by a three-beam

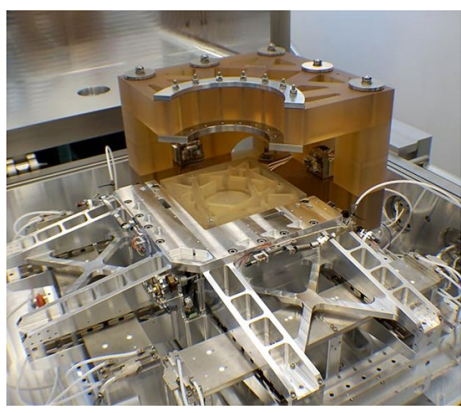

Fig. 2 Machine table and metrological Zerodur@ frame of the NPMM-200, without probing system [38] interferometer probing the $z$-mirror of the mirror corner, resulting in an enormous angle resolution of 0.00003 [36].

Experiments with the NPM machines revealed that in addition to the measuring capabilities, their positioning properties are excellent as well. The machines have an enormous position resolution of down to $5 \mathrm{pm}$, an ultra low position noise, trajectory deviations in the single nanometer range, and virtually no overshoot during changes in direction or when approaching a certain position $[36,39]$. Therefore, the NPM machines of the Technische Universität Ilmenau are perfect tools for the fabrication of 2D and 3D nanostructures in the size of a wafer.

For the described NPM machines, the focus was directed to a large range of motion in the $z$-direction for 3D measurements of highly curved objects (aspheres, mirrors) or step height standards, etc. However, tip-based nanomeasuring and nanofabrication increases demands regarding position stability and dynamic behavior in the $z$-direction. Therefore, a machine type, which especially addresses the requirements of this technology, was developed.

The NFM-100 is based on the same design principles as the NMM-1 and the NPMM-200, however, it is a $2 \mathrm{D}$ machine with a planar driving and guiding system. The absent $z$-axis results in a remarkable stability of the machine table in this spatial direction, which makes the NFM-100 a perfect platform for tip-based processes. The necessary $z$-movement in this machine is covered by the measuring/ tooling system and can be realized with a superior dynamic compared to the larger and heavier machine table of the
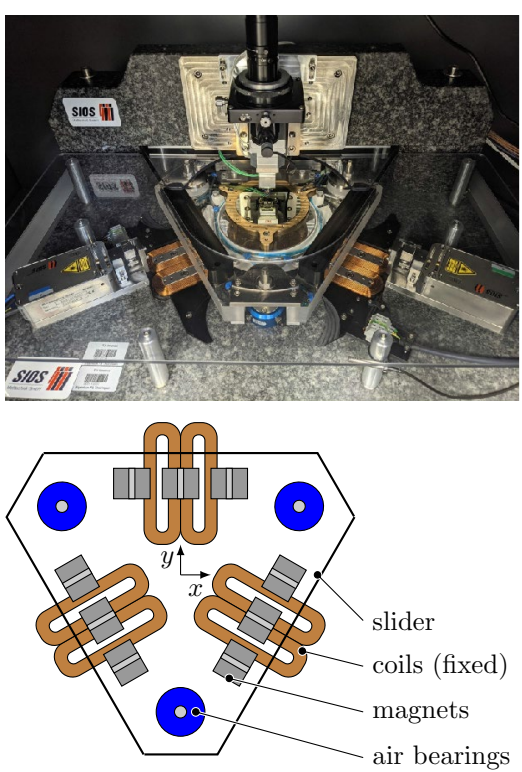

Fig. 3 Top: Photo of the NFM-100 with its tip-based measuring system. A 4"-wafer is placed on the machine table to illustrate the scale. [40] Bottom: Schematic illustration of the driving system arrangement of the NFM-100 [41] 
other NPM machines due to its low mass (Fig. 3). The NFM100 uses a planar direct driving system, where the single actuators are arranged in a common plane in a $120^{\circ}$ shape. The guiding of the machine table is realized by air bearings floating on a granite base. This setup results in a circular moving range of $\varnothing 100 \mathrm{~mm}$.

Table 1 gives a brief summary of the main parameters for the NMM-1, NPMM-200, and NFM-100.

\section{Challenges and Objectives of Modern Nanofabrication}

Deducing from the described deficits above, the question of transferring new nanofabrication technologies to large surface areas, as well as the question of a highly accurate, highly dynamic, and at the same time reproducible and longterm stable nanopositioning have not been answered so far. Thus, there is a great need for research into the flexible, cross-scale, and at the same time cost-effective production of complex nanoscale structures, even in smaller quantities. However, nanofabrication on large scales leads to completely new challenges in terms of speed, highly dynamic positioning, and achievable throughput, as well as the longterm stability of nanometrological equipment.

Therefore, it is necessary to advance into the border areas of nanostructuring with promising processes, which is not possible without using the unique advantages of the nanopositioning and nanometrology developed in Ilmenau. Furthermore, new requirements for nanopositioning and nanometrology are to be formulated from the needs of large-scale, cross-scale 3D nanofabrication techniques. In turn, solutions for a new quality of multi-axial, multi-tool nanofabrication machines, combined with new nanofabrication strategies, are to be derived. As a result, from the combination of nanofabrication and nanometrology on one machine, an immense advantage can be expected.

The central research question is to what extent the combination of advanced cutting-edge and laser-based processes

Table 1 Overview of the main parameters for the NPMs at the Technische Universität Ilmenau

\begin{tabular}{llll}
\hline & NMM-1 & NPMM-200 & NFM-100 \\
\hline Working range & $25 \times 25 \times 5$ & $200 \times 200 \times 25$ & $\varnothing 100 \times 0$ \\
$(\mathrm{~mm})$ & {$[31]$} & {$[35]$} & {$[40]$} \\
Resolution & 0.1 & 0.005 & 0.005 \\
$(\mathrm{~nm})$ & {$[32]$} & {$[36]$} & \\
Meas. uncert. & $<3$ & $<30$ & to be specified \\
$(\mathrm{nm})$ & {$[32]$} & {$[36]$} & \\
Tools & Manifold & Manifold & AFM, FE-SPL \\
& {$[42]$} & {$[27]$} & {$[40]$} \\
\hline
\end{tabular}

with nanopositioning and nanometrology will lead to new synergetic approaches of 3D nanofabrication that will extend the current limits of nanotechnologies and introduce new possibilities. The overall objective of the project can be split in certain research topics, derived from the requirements of nanotechnologies:

- Techniques for (AFM-) tip-based, cross-scale nanofabrication up to the limits of technical structurability in the sub-10-nm range on large surfaces, with the demand for the highest dynamics with the highest structuring accuracy.

- Techniques for laser-based nanofabrication at the physical limits of optical technologies, especially with regard to minimizing the lateral resolution of inclined surfaces.

- New solutions of nanofabrication on (non-planar) freeform surfaces with consideration of the interactions of the tools on these surfaces.

- Consistent application of metrological, error-theoretical strategies, such as error-minimal measurement principles and other principles of precision metrology, to nanofabrication techniques.

- Development of solutions for high-level parallelization of nanotools.

The research focus and results in these topics are described in more detail in the following sections.

\subsection{Laser-based Nanofabrication}

Methods for micro- and nanofabrication using mask-less lithography on (non-)planar surfaces, in combination with the outstanding metrological properties of the Technische Universität Ilmenau NPM machines, offer a unique opportunity for the realization of powerful large-area structuring. The main advantage of mask-less lithography is the virtually unlimited flexibility in the design of the exposure patterns.

From the point of view of laser nanoprocessing technologies, interference lithography and SNOM processes are important candidates for optical nanostructuring on freeform surfaces. This is especially true under the aspect of increasing lateral resolution and parallelizing structuring processes. The two-photon polymerization technique has experienced enormous progress in recent years [43]. Development of these processes and the combination with the approaches of NPM technology should enable completely new possibilities of cross-scale, complex 3D, optical material modifications of the highest spatial resolution and highest structuring accuracy.

Non-linear direct laser writing, also called two-photonbased direct laser writing, enables the mask-less 3D structuring of photosensitive materials. Two-photon-based direct laser writing combined with large-area and high-precision 
nanopositioning has already been demonstrated [44]. For the first time, structure widths far smaller than the diffraction limit can be realized with conventional two-photon laser writing in a positive photoresist, which was previously only possible with STED-inspired laser lithography. In the direct laser writing process, the focus of an ultrashort pulse laser is moved along $3 \mathrm{D}$ trajectories through the photosensitive material, which causes a spatial modification of the material along these trajectories by two-photon polymerization (2PP). Compared to single-photon polymerization (1PP), 2PP has a significantly smaller affected volume at the focal point of the laser beam, which enables the writing of structures in the nanometer range. To achieve the necessary high photon densities, a short-pulse laser is used, which increases the absorption probability compared with a continuous-wave laser. This also enables necessary high writing speeds [45] on the order of magnitude of $\mathrm{mm} \mathrm{s}^{-1}$, which are required to write structures over several $\mathrm{mm}^{2}$ in an acceptable time. In addition to the $2 \mathrm{PP}$ technique, the 1PP technique is also investigated using a common optical setup in which the corresponding laser sources can be coupled in separately. Figure 4 shows the setup of the NMM-1, equipped with a femtosecond laser. Structuring results generated with this setup, proving the potential of the utilized technique, are depicted in Fig. 5.

During the writing process, it is essential that the surface of the sample is always in the focal point of the writing laser to achieve minimum structure widths. For the detection of the sample surface, a laser focus sensor (LFS) was also

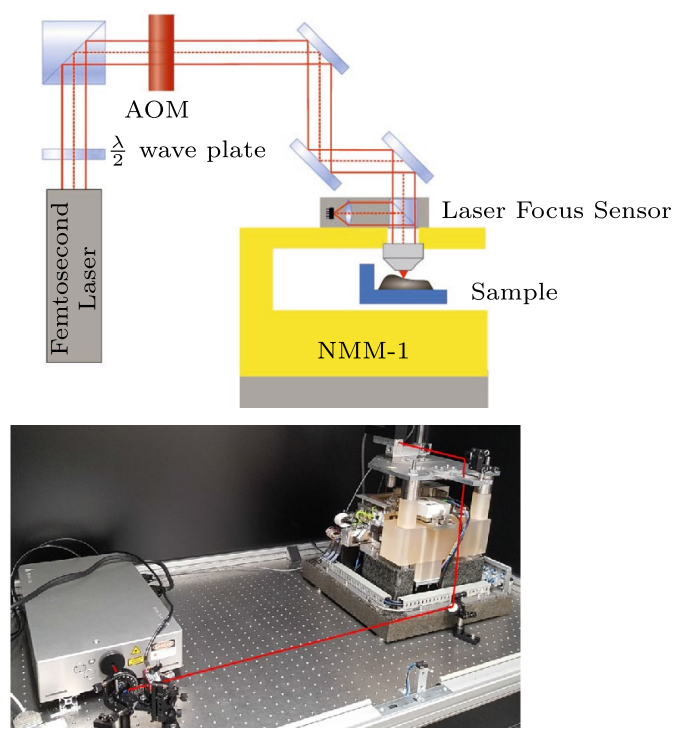

Fig. 4 Setup of the NMM-1 and the femtosecond laser for 2PP direct laser writing. The writing laser is switched by an acousto optical modulator (AOM) according to the structures to be written. A laser focus sensor is utilized to keep the surface of the sample in the focus spot of the writing laser
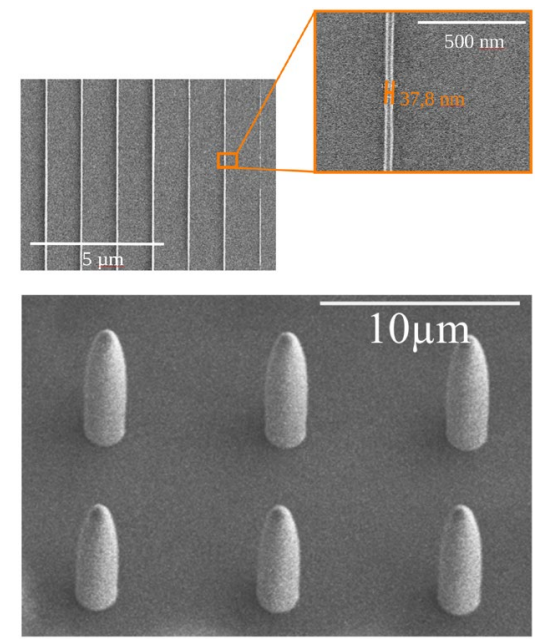

Fig. 5 Structuring results with the described setup. Top Smallest line widths of $37.8 \mathrm{~nm}$ could be achieved. Bottom Demonstration of the capability for 3D writing [46]

integrated into the optical setup, which allows for simultaneously detecting the position of the sample and structuring the applied resist material. Because the three systems, 1PP, 2PP, and LFS, work with different wavelengths, the partial beams can be guided through the optical setup without interfering with each other [47].

Table 2 summarizes the properties and challenges of laser-based nanofabrication processes.

\subsection{Tip-based Nanofabrication}

Regardless of the advantages of conventional optical fabrication techniques, there is a fundamental limit to the minimum distance of the structures written because of the optical diffraction limit. Thus, tip-based fabrication is a promising technology to manufacture features smaller than a characteristic size of $10 \mathrm{~nm}$. Current solutions for AFM tip-based nanofabrication are usually limited by structuring ranges in the micrometer range [7, 23, 25]. At the same time, the challenges with respect to precision, reproducibility, longterm and short-term stability, dynamics and others are well known [7, 24]. As a result, there is a need to research the combination of these nanofabrication technologies with adequate nanopositioning and nanometrology for a flexible, cross-scale fabrication of complex nanoscale structures. Here, the focus is directed to the connection of the process for "scanning proximal probe lithography" [48], which was also developed at the Technische Universität Ilmenau, with the NPM technology. With this combination, fundamental questions about the limits of structurability down to the molecular and atomic level are explored with simultaneously increasing processing fields of $1 "{ }^{\prime \prime}-8^{\prime \prime}$ and high speeds. Finally, new methods to increase throughput, such 
Table 2 Overview of the pros, cons, and challenges of laser-based nanofabrication

\begin{tabular}{l} 
Pros \\
High writing speed \\
Contactless \\
3D structuring (2PP) \\
Cons \\
Limited resolution \\
Point-wise writing \\
Challenges \\
Improve resolution (non-linear writing, material threshold) \\
Track surface, control working distance, focal spot size \\
Parallelization (multi-beam) \\
Dose control during non-uniform speed \\
\hline
\end{tabular}

as micro-stamping approaches, parallelization of AFM tips, as well as the further development of mix and match strategies, are essential.

At the Technische Universität Ilmenau, the scientifictechnical bases for novel, piezoresistive, thermomechanically actuated lithography cantilevers [49] and cantilever arrays $[50,51]$ were introduced and a unique understanding regarding their use for AFM and scanning probe lithography was gained. This work culminated in the development of special systems for very fast lithography and AFM measurements.

The fundamental elements for those techniques and achievements are self-sensing and self-actuated microcantilevers. The actuation is realized by the deposition of a meander-shaped aluminum layer on the silicon cantilever. When an electrical voltage is applied to this meander, it will heat because of its ohmic resistance. The bimorph effect will lead to a bending of the cantilever beam because of the different coefficients of thermal expansion of the cantilever layers. When applying a periodic voltage, a resonance at the inherent frequency of the cantilever can be stimulated. The integrated actuation spares the need for an external excitation, e.g. by a shaker piezo. This entails important advantages such as compact design, precise excitation of the cantilever at its resonance frequency, and the fast deflection of the cantilever at lower frequencies [30].

A piezoresistive measuring bridge sensing element is used to detect the actual deflection of the microcantilever. This assembly is placed at the clamping point of the beam. With the self-sensing cantilever, there is no need for an external deflection measuring system such as detecting the deflection of a laser spot with a four-quadrant photo diode (optical lever). This allows for a compact design and flexible operation of the complete system (Fig. 6).

The described cantilevers can be utilized in classical AFM mode for the pre- and post-inspection of samples
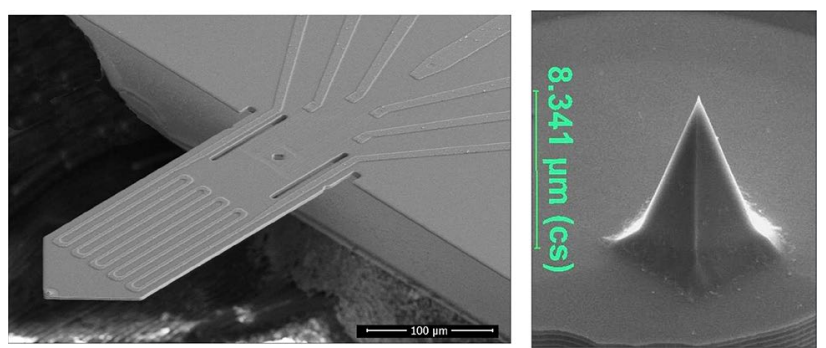

Fig. 6 Secondary electron images of an active cantilever with a piezoresistive read-out and a meander-shaped thermomechanical actuator (left) and magnification of the silicon tip of the cantilever (right) (courtesy nano analytik GmbH, Ilmenau, Germany) [40, 52]

during subsequent nanofabrication steps. Furthermore, there is the possibility of using them for nanostructuring with field-emission scanning probe lithography (FESPL, see next paragraph). These two modes of operation allow the sample to be measured and structured without changing tools, which is an enormous advantage as the positional relationship between successive processing steps is always maintained.

The FESPL technique for nanofabrication is based on a Fowler-Nordheim electron emission and is capable of structuring features in the sub-10-nm range under standard conditions $[30,53]$. Therefore, the technique can serve as a technology for prototyping sub-10-nm high-performance electronic devices $[54,55]$. In this mode of operation, the distance between cantilever tip and sample is closed-loop controlled to create a constant current between the cantilever tip and the surface. As a result, the resist is exposed by the electron current, allowing for tip-based lithography [56].

In addition to the plain fabrication of structures in the 10-nm range, the challenges of cross-scale structuring up to the macro-scale on the order of several $\mathrm{mm}^{2}$ are subject to current research at the Technische Universität Ilmenau. The lifetime of the cantilever tip is a severe problem in this application. Structuring 4" wafers with a 100-nm line distance results in an overall machining route of $81 \mathrm{~km}$ in length. Obviously, tip wear is an important factor for this kind of application.

For that reason, different tip materials are investigated in the current project. The diamond cantilever tip favored here, processed using a focused ion beam (FIB), exhibits long-term stability that is ideally suited for the structures to be written. This type of cantilever tip is suitable for writing processes over several hours to days because of the outstanding mechanical strength and the stable electron emission, which are necessary for large-area structuring. The FESPL technique was able to successfully produce structures with a width (half-pitch) of $15 \mathrm{~nm}$ [57]. In addition to the advantageous mechanical and tribological properties, diamond is also chemically inert, has a high thermal conductivity, 
and a high breakdown voltage. In nanocrystalline form, diamond can act as a good electron emitter when the grain boundary states have a relevant influence, or when electrons move along the surface. These and the previously described properties enable the successful generation of nanometer structures with FESPL in an ultra-thin calixarene layer with a significantly longer tip lifetime than conventional silicon cantilever tips.

A picture of the diamond tip and structured double line feature is shown in Fig. 7.

Electronic circuits based on established FinFETs technology are approaching a 3-nm node. Scanning probe lithography (SPL) is also contributing to "atom electronics", which will likely be the final step of electronics miniaturization, where electronic devices are realized by a single or few atoms. A pierced hollow cantilever tip can be used as a dynamic nanostencil mask and employed in the implantation of single dopant atoms. In this manner, every single dopant can be placed with precision and realize atomic-scale nodes [59].

So far, four NMM-1 nanopositioning and nanomeasuring machines with a measuring range of $25 \mathrm{~mm} \times 25 \mathrm{~mm} \times 5 \mathrm{~mm}$ and one NPMM-200 $(200 \mathrm{~mm} \times 200 \mathrm{~mm} \times 25 \mathrm{~mm})$ are available for the research work on future nanofabrication techniques. Shortly, an important large-scale device, the $\varnothing 100 \mathrm{~mm}$ nanofabrication machine NFM-100, was added to this equipment base. This machine was built by the SIOS Meßtechnik and is based on three basic techniques developed at the Technische Universität Ilmenau, which determine the state of the art [41]. With the NFM-100, the RTG is now in a position to combine the advantages of SPL with the outstanding properties of nanopositioning technology, entirely in line with the outlined central research idea.

The application of large area AFM scans and FESPL is currently investigated using the NFM-100. There, the large positioning range of this machine was utilized to successfully demonstrate long-range AFM scans over $50 \mathrm{~mm}$ and

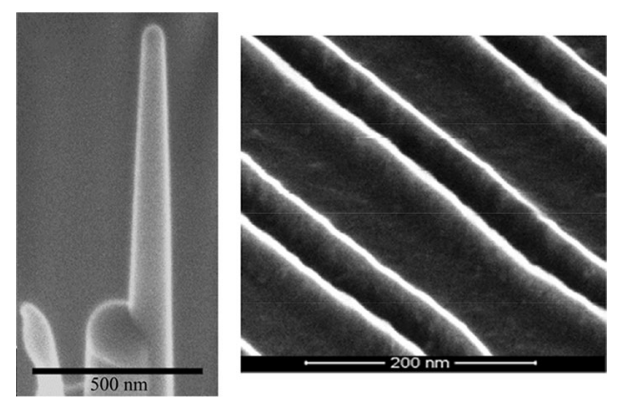

Fig. 7 Left: Diamond tip for FESPL after micro-machining using a focused gallium ion beam [58]. Right: SEM image of double line features, structured with the described FESPL technique. A fully etched trench between the two parallel lines is observed and the line distance is $30 \mathrm{~nm}$ [56] area scans of $0.5 \mathrm{~mm} \times 0.5 \mathrm{~mm}$. Furthermore, the overall resolution of the combined system and first results in structuring calixarene coated samples could be shown [40,41] (Fig. 8).

Table 3 summarizes the properties and challenges of tipbased nanofabrication processes.

\subsection{Areal Nanofabrication}

As described in the paragraph before, point-wise structuring of large areas is a time-consuming process. For this reason, several writing techniques capable of parallel, respectively two-dimensional, exposure have been investigated.

Interference lithography is a powerful method for fast large area exposure of micro- and nanostructures. Compared with the direct-writing methods of laser or electron beam lithography, the decisive advantage is the possibility of simultaneous exposure of 2D areas of the substrate. Optical effects such as the Talbot effect have been used to create periodic structures in optical lithography [60]. The projection on curved surfaces requires illumination with free-form wave fronts, which are usually generated by phase modulation. However, because of the coherence of the used laser source, parasitic speckle effects occur, which result in an increased roughness of the exposed structures.

Therefore, the use of incoherent illumination is investigated to use the LAU effect to realize optical lithography on
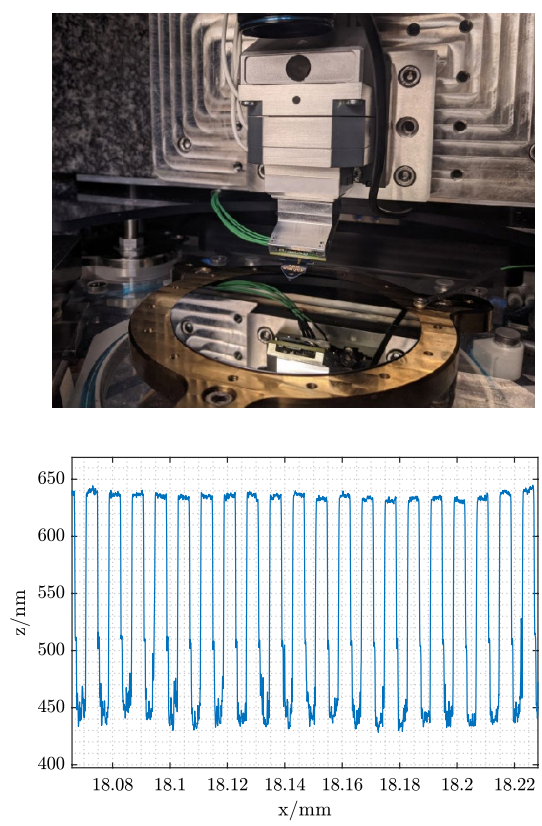

Fig. 8 Top: Close-up view of the NFM-100 machine table and mounted tip-based AFM/FESPL system. Bottom: Section of a line scan of a $8 \mu \mathrm{m}$ pitch sample, acquired with the described AFM system and NFM-100. Total scan length was $50 \mathrm{~mm}$ and scan velocity was $20 \mu \mathrm{m} \mathrm{s}^{-1}[40]$ 
Table 3 Overview of the pros, cons, and challenges of tip-based nanofabrication

\begin{tabular}{l} 
Pros \\
Single-nm resolution \\
Self-actuated and self-sensing cantilevers \\
Pre- and postinspection by same tool \\
Cons \\
Low writing speed \\
Tip wear \\
Challenges \\
Parallelization (multi-cantilever) \\
Mix and match \\
Increase tip lifetime and/or reproducibly change tip \\
\hline
\end{tabular}

flat and curved surfaces and to increase the lateral resolution through cross-correlation between periodic rectangular intensity distributions. Here, substrates with complex surface topologies pose a particular challenge. To face the described challenges, optical system solutions for interference exposure were developed. First, the scalar diffraction theory was used to mathematically describe and simulatively investigate the light propagation in the LAU experimental setup under spatially coherent and spatially incoherent illumination. Subsequently, a corresponding setup was realized. Previously unknown effects in the generation of the interference pattern could be revealed during the investigations of the LAU effect. Finally, the LAU fringes generated with the developed setups were used in optical lithography on planar and curved surfaces (Fig. 9).

Holographic projections by amplitude modulating spatial light modulators (SLM) are capable of planar exposures with structure sizes less than $1 \mu \mathrm{m}$ [62]. These systems are limited in their depth of field and are only suitable for structuring non-planar surfaces to a limited extent. The depth of field can be increased by using phase-only liquid crystal on silicon (LCoS) SLM. The implementation of such an SLM system was another push for the application of this technique. For that purpose, in-depth investigations regarding the hologram calculations and adaptation to $3 \mathrm{D}$ surfaces were conducted, starting from simple planar exposure. The developed algorithms were used to simulate the projection quality for various parameters and compare the variants in terms of quality, intensity, and diffraction efficiency. Subsequently, a corresponding experimental setup was realized to verify the simulation results. Various design-related effects of the LCoS-SLM, such as crosstalk between neighboring pixels and the limited pixel fill factor that can lead to disturbing diffraction efficiency, were identified, investigated, and compensated for where possible.

Nanoimprint lithography (NIL) is a low-cost, areal patterning process that offers the high throughput and

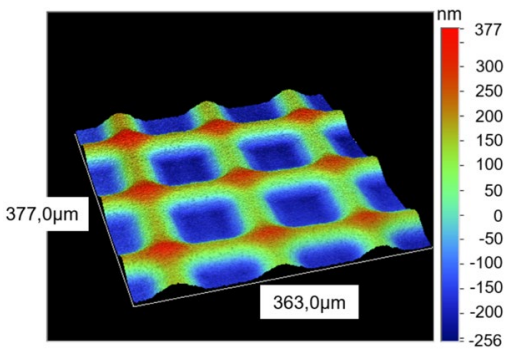

Fig. 9 Demonstration of lithography by LAU fringes on a planar glass wafer [61]

resolution needed for nanofabrication. Since the process is purely mechanical, there are no limitations because of optical effects such as scattering, refraction, and interference, as in common photolithographic techniques. The process uses a flexible, transparent stamp and resists curing by UV light (UV-NIL). The challenge with any imprint process is the positioning and alignment of the stamp relative to the sample. Here, the outstanding positioning capability of the NPM machines is an invaluable advantage. Exact alignment to existing structures (overlay) or during a stitching process is ensured at any time because of the position uncertainty in the single-digit nanometer range. The trajectory fidelity of the NPM machines also plays a decisive role, such that the stamp can be approached perpendicularly to the sample surface and no unacceptable transverse movement occurs, which would affect the final imprint outcome. The results of an imprint process on a flat sample show achievable lateral resolutions on the order of $100 \mathrm{~nm}$ [63]. For structuring strongly curved surfaces with local inclinations of up to $45^{\circ}$, it is necessary to incline the stamp itself. The challenge here is to approach the stamp perpendicularly to the surface of the sample to produce an error-free impression. The realization of a corresponding setup was conducted using the five-axis NPM machine described in Sect. 4.4. A combination with the manufacturing processes from the Sects. 4.1 (direct laser writing) and 4.2 (FESPL) could also be demonstrated by realizing a mix-and-match process. This verification proves the potential of the NPM machines for a synergy of different structuring technologies, where a master for the NIL process is generated with comparatively slow point-based processes and then duplicated in a time- and cost-efficient manner (Fig. 10).

The successful realization of a complete process chain for the production of nanostructures on large surfaces represents a significant step in the alternative manufacturing of nanostructures with the NPM machines of the Technische Universität Ilmenau. The different manufacturing processes - tip-based nanolithography, direct laser writing, and nanoimprint lithography — have been successfully combined in such a way, that time- and cost-efficient series production 


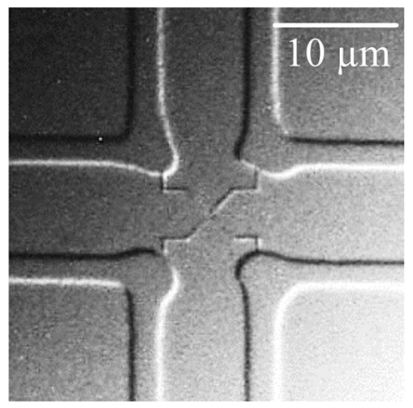

Fig. 10 NIL stamp, fabricated by mix and match of DLW and FESPL and subsequent replication of the written master features into a negative PDMS structure [54]

of complex structures is possible. Using a mix-and-match approach, individual parts of the structure to be produced can be written with the optimal process for the particular feature and then reproduced using NIL. This replication means that the comparatively slow point-based writing processes are only necessary once, and the complex structure can then be reproduced several times, thus producing complete structures on large areas at high resolution.

Table 4 summarizes the properties and challenges of areal nanofabrication processes.

\subsection{Nanofabrication on Free-Form Surfaces}

The functionalization of surfaces with nanostructures has enormous potential for the development of products with previously unknown properties and is the subject of current international research initiatives. The research focus is currently limited to flat surfaces. The functionalization of non-planar surfaces (in optics, e.g., aspheres and freeform surfaces) using nanostructures would enable further significant technical progress [64]. In addition to the

Table 4 Overview of the pros, cons, and challenges of areal nanofabrication

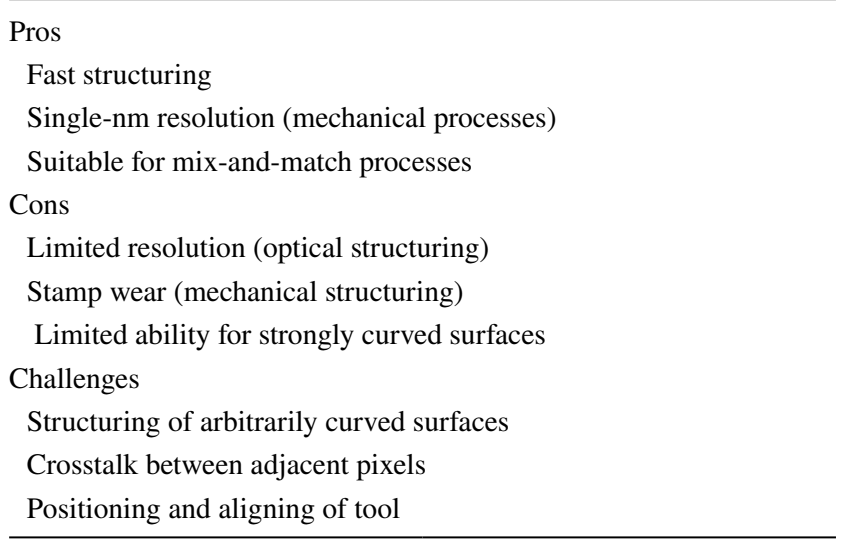

challenges of mechanical and optical structuring, there are metrological and technological requirements for the NPM machines resulting from the complex task. The aim is to show that nanostructuring is also feasible on inclined, arbitrarily shaped surfaces and that it is possible to connect the produced 3D structures with nanometer precision to precision components by applying the NPM technology. This can introduce functional integration of photonic/plasmonic systems on macroscopic free-form surfaces with new properties $[65,66]$. This approach is associated with the need for additional movements to address features in $3 \mathrm{D}$. In addition to a theoretical penetration, new metrological and precision mechanical approaches, combined with new control concepts and machining strategies, have to be developed. These must fulfil the combined metrological requirements of measurement and machining in terms of production technology.

The majority of NPM machines are based on three linear traversing axes arranged in a Cartesian coordinate system. However, these reach their limits on samples with strong local inclination angles, undercuts or large aspect ratios. A complete measurement or structuring with nanometer precision of such a specimen in a single clamping is virtually impossible with this restricted design. Alignment of the tool main axis normal to the curved surface enables compliance with the precision requirements. By integrating additional rotational axes, the accessibility of demanding geometry elements can be significantly improved without disadvantageous modifications of the probing system. At the same time, the necessary expansion of the kinematic freedom must not compromise the precision properties of the original NPM machine. Therefore, the systematic development of multi-axis machine structures with up to six degrees of freedom with large ranges of motion and ensuring the metrological validity of the overall concept was a focus of the project. For this purpose, the design fundamentals for multiaxis nanopositioning machines, the integration of additional rotational axes for positioning the measuring or processing tool to the surface of the sample, were deeply investigated $[67,68]$. Subsequently, the optimal structure for the set of requirements was searched among a large number of functional variants. Based on the systematic development and extensive preliminary investigations, two rotational axes for the additional degrees of freedom were designed for integration into the NMM-1 of the Technische Universität Ilmenau. The following picture shows the functional concept of the chosen attempt.

The developed configuration was implemented (Fig. 11) and the metrological performance was comprehensively investigated. In addition, as proof of functionality, a measuring tool (laser focus sensor) and a processing tool (NIL stamp, Sect. 4.3) were integrated into the NMM-1. Figure 12 shows the integrated NIL stamp and the imprint results for an inclination of $0^{\circ}$ and $45^{\circ}$. 


\subsection{Dynamics and Traceability}

With a suitable controller design, it is possible to tune the guidance behavior of an NPM machine largely independent of the disturbance behavior. High demands on the performance of the disturbance compensation require suitable models for the disturbance influence; however, the smallest delay is required in the reaction of the controller to the disturbance. Therefore, new strategies for real-time control and disturbance models were developed for the purpose of highly dynamic active disturbance compensation in probing and machining systems. These structural specifications were the basis of the design of powerful adaptive control concepts. The focus here was on the application of non-integer, linear, time-invariant systems [70]. This concept is used in control engineering because of the increased degree of freedom in the frequency domain [71]. How the change in the order of the non-integer derivative affects the estimation of the system states was investigated. Based on a system description adapted to the NPM machines, observers can be designed, which converge faster.

This attempt is well suited for describing real processes with memory. The representation of classical systems of integer order with the aid of non-integer operators allows observers to be designed [72], which achieve increased convergence rates despite sensor noise. However, preliminary work showed deficits in the modeling with regard to the steady-state accuracy of the models, in particular static hysteresis. This in turn leads to only poorly identified highfrequency model components (e.g., resonances or creep behavior [73]).

Detailed modeling, especially of hysteresis phenomena, was necessary to improve the model quality and thus also the control. Operator-based or differential approaches (and also fractional approaches [74]) are currently being compared with regard to their structure, complexity, and real-time capability. Furthermore, sensor quantizations, which so far have simply been regarded as disturbances, are included, as they become relevant for the targeted accuracies.

For highly dynamic control of the tip- and laser-based machining processes, the current between cantilever and substrate (Sect. 4.2) or the laser power (Sect. 4.1) must be actively controlled in addition to the distance if the surface properties are unknown. For this purpose, a forward prediction of the geometry to be addressed next has to be derived from the passed trajectory using observer approaches. If the unknown geometry is modeled as a disturbance, fractional approaches can help to expand the possible disturbance class. Multidimensional approaches are required to consider directionality. Subsequently, the control of the cantilever current or laser power is to be coupled with the subordinate position control.

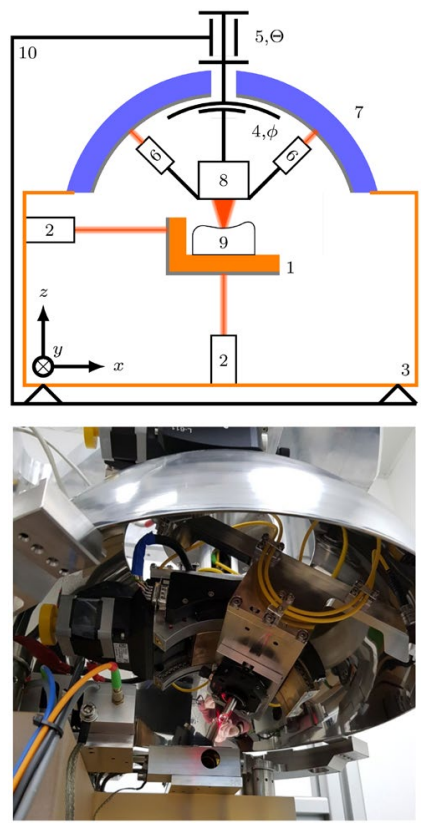

Fig. 11 Top: Metrological setup of the five-axis Nanomeasuring Machine NMM-1 (2D visualization; only 2 of 3 interferometers and distance sensors are shown.). 1 mirror corner (moving in $x, y, z) ; 2$ interferometers; 3 metrological frame; 4 goniometer axis; 5 rotational axis; 6 distance sensors of the reference system; 7 reference hemisphere; 8 tool; 9 measuring object; 10 force frame [69]. Bottom: Photograph of the designed setup for the two additional rotational axes

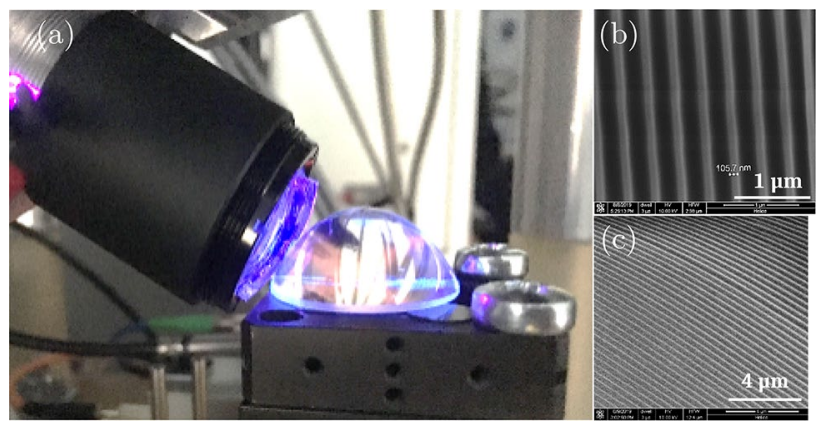

Fig. 12 Nanoimprint on a plano-convex lens: a Experimental setup with lens on an NMM-1 machine table and NIL stamp, inclined by $45^{\circ}$ to the $z$-axis, $\mathbf{b}$ image of imprint result on the top of a sample (imprint at $0^{\circ}$ ) and $\mathbf{c}$ image of imprint result at the edge of a sample (imprint at $45^{\circ}$ ) [69]

The second approach to increase the convergence of an observer is to represent integer linear time-invariant systems by non-integer operators. The use of fractional derivatives in the observer design leads to increased convergence in the time interval immediately after the initial time point. In this context, the periodic cancellation of the non-integer dynamics leads to exponential stability. The theoretically developed controller structures were verified in an experimental setup. 
A current problem in nanopositioning is the robustness of the control systems, as they are usually very susceptible to disturbances and uncertainties. Therefore, for control with 6 degrees of freedom, an adaptive control theory is developed for the interconnection and damping assignment passivity-based control (IDA-PBC) methodology in the implicit frame. Passivity-based control (PBC) plays a central role in the development of algorithms that exploit the passivity and energy characteristics of mechanical systems. Moreover, the IDA approach shapes the system energy, i.e., the kinetic plus potential, through feedback that maintains the closed-loop mechanical structure. The novelty of this adaptive control approach is to asymptotically stabilize a class of mechanical systems even under constrained disturbances and uncertainties. IDA-PBC is a well-established robust technique and the adaptive term improves the compensation of limited disturbances and uncertainties. The applicability of this novel control concept in highly dynamic nanopositioning was investigated and serves as a basis for identifying improvements in the drive arrangement of NPM machines based on simulations and measurements.

Highly dynamic nanopositioning in the vertical direction is a particular issue in nanofabrication. Exact movements in this direction pose an exceptional challenge because of the gravitational force: the mass of the vertically moving parts must be permanently held in the target position by the drive system against the force of gravity, which usually results in continuous power consumption of the associated drives and an according heat dissipation. Therefore, an integrated lifting and actuating unit (LAU) was developed that addresses this challenge and enables positioning over large ranges of motion with nanometer resolution while minimizing power consumption. The weight force in vertical axes is usually compensated by pre-tensioned springs or pneumatic cylinders. The use of springs requires an adjustment of the spring characteristic curve to generate a constant compensation force over the entire stroke of the vertical axis. Furthermore, additional degrees of freedom are required in the corresponding spring mechanism for different loads, e.g., because of different measurement objects. Conventional pneumatic cylinders suffer from the friction between piston and wall of the bore, which leads to the known stick-slip-effect and makes high-resolution positioning nearly impossible. For this reason, a special pneumatic weight force compensation was developed where the compensation force can be easily adapted to different loads by varying the pressure in the pneumatic chamber. Therefore, only the forces for positioning (acceleration) and compensation of external disturbances are generated by the actual driving system, reducing the power consumption to a minimum [75]. The unique detail is the frictionless construction of the pneumatic cylinder with a pneumatic sealing, realized by a radial air bearing. Virtually unlimited position resolution is within reach because of the pure fluidic friction (Fig. 13).

Forces in AFM-based nanofabrication technologies and corresponding material properties have a great influence on the wear of the AFM tip. Therefore, the effects of machining forces on tool behavior and position in nanofabrication were investigated, with a particular focus on achieving traceable calibration of combined force-position sensor systems. The focus was on cantilevers for tip-based nano-measurement and structuring, whose probing force is proportional to the deflection. The stiffness has large uncertainties because of manufacturing tolerances and other influences and must therefore be calibrated to ensure clearly defined measuring and manufacturing parameters.

The aim of the work was to significantly improve the metrologically traceable calibration of force sensors in the nanonewton range and develop a fundamental measuring device for small forces. This fundamental measuring device is to be traced back to SI units in three different ways:

- Gravimetrically with small mass standards

- Electromagnetically according to the Kibble balance principle

- Electrostatic

A further goal is the transfer of the calibration of nanonewton forces to MEMS systems. In context with the tip-based technologies (Sect. 4.2), methods for direct and indirect calibration of the self-actuated MEMS probes have to be identified and are currently being investigated. The long-term goal is the development of MEMS probes that measure forces and displacements and are self-calibrating in situ [78]. With both the cantilever calibration system and calibrated cantilevers/ probes, the implementation and investigation of calibrations on reference marks of known geometries and material properties on the object itself are targeted, taking advantage of the high positioning accuracy of the NPM machines.

Cantilevers must be calibrated with forces in the range of nanonewtons because of the risk of damage or deformation, which results in increased measurement uncertainty. Thus, a load cell whose mechanics and measuring range are optimized for the calibration of cantilevers is required. The evaluation of the metrological quality and its adaptation to the parameter requirements of nano-machining were the focus of the research. With the calibration procedure developed, the necessary low measurement uncertainties can be achieved and the calibration process corresponds to the deformation of the cantilevers in the subsequent application. To reduce the uncertainty contribution of the force measurement, a new load cell was developed (Fig. 14) that overcomes the aforementioned limitations and problems.

With this completely new cantilever calibration system, the calibration of a soft cantilever $\left(c=19 \mathrm{mN} \mathrm{m}^{-1}\right)$ with a 


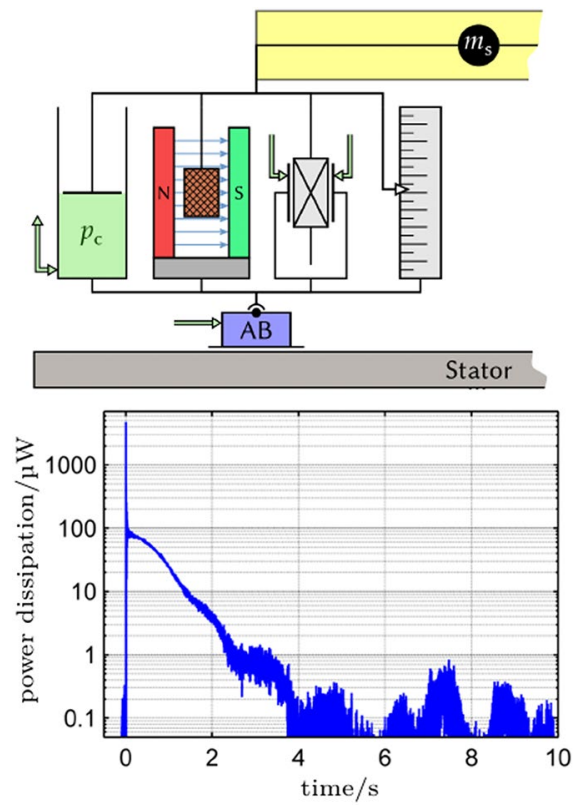

Fig. 13 Top Principle of the LAU, with the components: pneumatic weight force compensation, precision Lorentz-force drive, aerostatic guiding, measurement system [76]. Bottom Power dissipation in the LAU for a jump of $1 \mu \mathrm{m}$ in $z$-direction with a velocity of $1 \mathrm{~mm} \mathrm{~s}^{-1}$ [77]

relative measurement uncertainty of $1.5 \%(k=2)$ can be achieved at a maximum calibration force of $100 \mathrm{nN}$ [79]. Subsequent SEM images of this cantilever showed no detectable tip wear.

For tactile measurements and especially machining, force-guided control of the NPM machines [80] is required in addition to pure position control. As a result, metrological traceability of the force vector measurement is crucial because knowledge of the feed force and position-dependent mechanical interaction between the sample and the tool is important for precise machining. Therefore, traceable calibration of suitable single- and multi-component force sensors is necessary. For this purpose, multi-component forcetorque sensors with a high measurement dynamic range, fast transmission properties, and purely electrical in situ calibration are developed and investigated. There, the focus is specifically on the measurement and control of torques for nanofabrication and the traceable realization for calibrations of very small torques. This results in high demands on the measurement uncertainty of the torque standard measuring devices. Thus, a new principle for setting different torques was developed based on the jockey-weight principle. The basic idea consists of a high-precision mass, which is moved along a lever and thus generates variable torques depending on its distance to the center of rotation. The mass is represented by a silicon sphere of $1 \mathrm{~kg}$ mass, which is a secondary standard with an extremely low relative standard uncertainty. The developed calibration principle was transferred

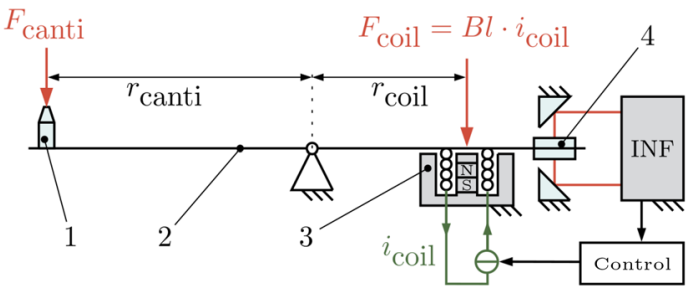

Fig. 14 Mechanical setup of the one-flexure load cell for traceable cantilever calibration: 1 point of load application; 2 monolithic balance lever; 3 actuator with magnet and coil; 4 measuring mirror of the differential interferometer (INF) [79]

to a corresponding experimental setup and comprehensively investigated. The focus was on the question of the transferability of static calibrations with comparative or fundamental methods to rapidly changing forces, which were investigated by model and experiment.

\section{Conclusions and Outlook}

The central research question addressed in this paper is how the latest nanofabrication techniques can be synergistically combined with the outstanding capabilities of nanopositioning and nanomeasuring (NPM) machines in such a way that new, innovative, cross-scale, large-area solutions for 3D nanofabrication become possible. The consistent application and extension of error minimized metrology principles to nanofabrication systems and the derivation and investigation of new nanofabrication opportunities and strategies should be realized. The vision is to create a powerful, universally applicable and viable nanofabrication platform that can effectively contribute to the advancement of next-generation nanotechnologies.

New and alternative nanofabrication processes have been the subject of research for several years [15, 81]. However, the processes are limited to basic investigations of new nanostructuring phenomena, which are usually demonstrated only on areas of a few square micrometers. The problem of applying efficient, highly accurate, highly dynamic, simultaneously reproducible and long-term stable nanopositioning to large surface areas for the purpose of nanofabrication has not been systematically investigated so far. This is where the RTG NanoFab steps in. The focus of the RTG is on researching new possibilities for innovative nanofabrication processes by combining them with highly developed approaches to nanopositioning and nanometrology.

The goal is to combine high-performance, tip- and laserbased micro- and nanofabrication techniques with the unique possibilities of nanopositioning and nanometrology developed at the Technische Universität Ilmenau to introduce completely new possibilities for nanofabrication. This way, 
the current technical limits of structurability can be overcome to realize structures with sub-nanometer precision and three-dimensionality across scales in large working areas (up to $200 \mathrm{~mm} \times 200 \mathrm{~mm} \times 25 \mathrm{~mm}$ ). The implementation of the International Roadmap for Devices and Systems (IRDS) [6] represents a significant technology driver. In addition to the increasingly complex optical lithography for highly integrated circuits, which only remains affordable through mass production, alternative nanofabrication techniques for flexible, universal, and iterative production are increasingly coming into focus. There is a research deficit in realizing such tip-based nanofabrication technologies at the limits of patternability $(1-5 \mathrm{~nm})$ on larger areas. Novel functionalizations of precision optics can be realized by transferring 2D/3D laser nanoprocessing technologies for sub-wavelength patterning to non-planar large-area surfaces. Finally, the combination of nanomeasurement and nanofabrication technologies in one machine can be used to develop new highly efficient nano-repair technologies. In this context, the consistent and systematic application of metrological approaches to (nano-) fabrication technologies represents an important innovative and unique research approach that makes it possible to create the kinematic and metrological prerequisites for 3D nanofabrication with new quality.

The uniqueness of the NPM technology developed at the Technische Universität Ilmenau, the combination of the highest resolutions with cross-scale measurement and positioning ranges (up to $10^{-10}$ ) introduces completely new technical possibilities.

In the future, optical-induced local thermal effects [82], which appear to have the potential for direct laser writing of sub-50-nm structures that are well below the diffraction limit, will be subject to research for the integration in NPM machines. This reinforces the goal of large-scale sub-wavelength patterning, from planar to curved and 3D surfaces, and from point-by-point to array-based processes for further work. The comprehensive inclusion of machining forces and the extension of the mechanical models to compliant systems will enable further development of highly dynamic, highly accurate, and high-resolution nanopositioning/nanofabrication. Finally, the demonstration of demanding applications is a target in the next few years. In particular, the interdisciplinary realization of process chains for the production of novel single-electron, photonic, and quantum components will be addressed.

The vision is to create the foundation for realizing comprehensive nanofabrication on the basis of NPM machines. The goal is also to systematically and consistently expand the field of nanopositioning and nanomeasuring machines with respect to the field of nanofabrication.

Acknowledgements This work was supported by the Deutsche Forschungsgemeinschaft (DFG) in the framework of the Research
Training Group Tip- and Laser-based 3D-Nanofabrication in extended macroscopic working areas (GRK 2182) at the Technische Universität Ilmenau, Germany.

Author Contributions Conceptualization: E. M., T. F., R. F., J. R., C. S., S. Si., S. St., R. T., L. Z., J.-P. Z., I. W. R.; Supervision: E. M., T. F., R. F., I. O., J. R., C. S., S. Si., S. St., R. T., L. Z., J.-P. Z., C. R., I. W. R.; Investigations and Realization of the measurements: X. C., 0. D., F. F., D. F., S. G., M. H., J. K., A. M., T. S., R. S., S. Su., L. W., C. W., C. R., U. B., J. S. + I. O.; Analysis of the data: X. C., 0. D., F. F., D. F., S. G., M. H., J. K., A. M., T. S., R. S., S. Su., L. W., C. W., C. R., J. S., I. O.; Writing - original draft preparation: I. O.; Writing - review and editing: E. M., J. S., T. H., I. W. R., C. S., R. T., X. C., 0. D., S. G., A. M., R. S., S. Su. L. W.;

Funding Open Access funding enabled and organized by Projekt DEAL.

Data Availability Statement All original data are available upon request from the corresponding author.Code Availability Software code is available upon request from the corresponding author, depending on the original software license/EULA.

\section{Declarations}

Conflict of interest The authors declare that they have no conflicts of interest.

Open Access This article is licensed under a Creative Commons Attribution 4.0 International License, which permits use, sharing, adaptation, distribution and reproduction in any medium or format, as long as you give appropriate credit to the original author(s) and the source, provide a link to the Creative Commons licence, and indicate if changes were made. The images or other third party material in this article are included in the article's Creative Commons licence, unless indicated otherwise in a credit line to the material. If material is not included in the article's Creative Commons licence and your intended use is not permitted by statutory regulation or exceeds the permitted use, you will need to obtain permission directly from the copyright holder. To view a copy of this licence, visit http://creativecommons.org/licenses/by/4.0/.

\section{References}

1. Fritze M (2021) Post-Moore: prospects for novel patterning of low volume ICs. In: Panning EM, Liddle JA (eds) Novel patterning technologies 2021. Vol. 11610. International society for optics and photonics. SPIE, Bellingham. https://doi.org/10.1117/12.2593229

2. Moore GE (2006) Cramming more components onto integrated circuits, Reprinted from Electronics, volume 38, number 8, April 19, 1965, pp.114 ff. In: IEEE Solid-State Circuits Society Newsletter 11.3, pp. 33-35. https://doi.org/10.1109/N-SSC.2006.47858 60

3. Bjorkholm JE (1998) EUV Lithography-The Successor to Optical Lithography? In: Intel Technology Journal 3, p. 98

4. Waldrop MM (2016) The chips are down for Moore's law. Nature 530(7589):144-147. https://doi.org/10.1038/530144a

5. Roadmap for Semiconductors (ITRS), T. I. technology. Executive Summary (2013) 
6. IEEE. International Roadmap for Devices and Systems (IRDS) (2020) https://irds.ieee.org/editions/2020 (visited on 02/25/2021)

7. Bloschock KP, Schofield AR, Kenny TW (2011) Tip-based nanofabrication: an approach to true nanotechnology. In: 8031. Ed. by George T, Islam MS, Dutta AK, pp 19-28. https://doi.org/10. $1117 / 12.887266$

8. Pronko P, Dutta S, Squier J, Rudd J, Du D, Mourou G (1995) Machining of sub-micron holes using a femtosecond laser at 800 nm. Opt Commun 114(1):106-110. https://doi.org/10.1016/00304018(94)00585-I

9. Koch J, Korte F, Fallnich C, Ostendorf A, Chichkov BN (2005) Direct-write subwavelength structuring with femtosecond laser pulses. Opt Eng 44(5):1-5. https://doi.org/10.1117/1.1904053

10. Sahoo S, Parveen S, Panda J (2007) The present and future of nanotechnology in human health care. In: Nanomedicine: nanotechnology, biology and medicine 3.1, pp. 20-31. https://doi.org/ 10.1016/j.nano.2006.11.008

11. Prasad R, Kumar V, Prasad KS (2014) Nano technology in sustainable agriculture: present concerns and future aspects. Afr J Biotechnol 13(6):705-713. https://doi.org/10.5897/ajbx2013.13554

12. Serrano E, Rus G, García-Martínez J (2009) Nano technology for sustainable energy. Renew Sustain Energy Rev 13(9):2373-2384. https://doi.org/10.1016/j.rser.2009.06.003

13. Diallo MS, Fromer NA, Jhon MS (2014) Nanotechnology for sustainable development: retrospective and outlook. In: Diallo MS, Fromer NA, Jhon MS (eds) Nanotechnology for sustainable development. Springer, Cham, pp 1-16

14. Manske E, Jäger G, Hausotte T, Füssl R (2012) Recent developments and challenges of nanopositioning and nanomeasuring technology. In: Measurement science and technology 23.7, 074001. https://doi.org/10.1088/0957-0233/23/7/074001

15. Tseng AA, Jou S, Notargiacomo A, Chen TP (2008) Recent developments in tip-based nanofabrication and its roadmap. J Nanosci Nanotechnol 8(5):2167-2186. https://doi.org/10.1166/jnn.2008. 243

16. Shamoun B, Chandramouli M, Liu B, Juday RK, Bucay I, Sowers AT, Abboud FE (2021) Multi-beam mask writer in EUV era: challenges and opportunities. In: Panning EM, Liddle JA (eds) Novel patterning technologies 2021. International society for optics and photonics, vol 11610. SPIE, Bellingham, pp 64-82

17. Hiura M, Takabayashi Y, Kimura A, Morohoshi H, Suzaki Y, Matsumoto T, Cherala A, Choi J, (2021) Nanoimprint performance improvements for high volume semiconductor device manufacturing. In: Panning EM, Liddle J (eds) A Novel patterning technologies 2021. International society for optics and photonics, vol 11610. SPIE, Bellingham. https://doi.org/10.1117/12.2584675

18. Stockman MI (2011) Nanoplasmonics: past, present, and glimpse into future. In: Opt. Express 19.22 , pp. 22029-22106. https://doi. org/10.1364/OE.19.022029

19. Xia F, Wang H, Xiao D, Dubey M, Ramasubramaniam A (2014) Two-dimensional material nanophotonics. In: Nature photonics 8.12, pp. 899-907. https://doi.org/10.1038/nphoton.2014.271

20. Nussinov R, Alemn C (2006) Nanobiology: from physics and engineering to biology. In: Physical biology 3.1. https://doi.org/ 10.1088/1478-3975/3/1/e01

21. Riehemann K, Schneider SW, Luger TA, Godin B, Ferrari M, Fuchs H (2009) Nanomedicine-challenge and perspectives. Angew Chem Int Ed 48(5):872-897. https://doi.org/10.1002/anie. 200802585

22. Nie Z, Yang S, Zhang X (2021) Plasmonic lithography: from superlens to hyperlens and recent metamaterial development. In: Panning EM, Liddle JA (eds) Novel patterning technologies 2021. International society for optics and photonics, vol 11610. SPIE, Bellingham. https://doi.org/10.1117/12.2583561

23. Durrani Z, Jones M, Kaestner M, Hofer M, Guliyev E, Ahmad A, Ivanov T, Zoellner J-P, Rangelow IW (2013) Scanning probe lithography approach for beyond CMOS devices. In: Tong WM, Resnick DJ (eds) https://doi.org/10.1117/12.2012199

24. Tseng AA (2011) Advancements and challenges in development of atomic force microscopy for nano fabrication. Nano Today 6(5):493-509. https://doi.org/10.1016/j.nantod.2011.08.003

25. Ballard JB, Owen JHG, Fuchs, E, McDonnell S, Dick D, Mordi G, Azcatl A, Seitz O, Campbell P, Veyan JF, Chabal Y, Wallace RM, Bischof M, Jaeger D, Reidy R, Sarkar N, Randall JN (2013) Atomically-precise three-dimensional top down fabrication. In: pp 764-767. https://doi.org/10.1109/Transducers.2013.6626878

26. Baró A, Reifenberger R (2012) Atomic force microscopy in liquid: biological applications general atomic force microscopy. Wiley, Hoboken

27. Jäger G, Manske E, Hausotte T, Müller A, Balzer F (2016) Nanopositioning and nanomeasuring machine NPMM-200a new powerful tool for large-range micro and nanotechnology. In: Surface topography: metrology and properties $4.3,034004$. https://doi.org/ 10.1088/2051-672x/4/3/034004

28. Vicary JA, Miles MJ (2009) Real-time nano fabrication with highspeed atomic force microscopy. In: Nanotechnology 20.9, 095302. https://doi.org/10.1088/0957-4484/20/9/095302

29. Tseng AA (2011) Tip-based nanofabrication. Springer, New York. https://doi.org/10.1007/978-1-4419-9899-6

30. Rangelow IW Lenk C Hofmann M, Lenk S, Ivanov T, Ahmad A, Kästner M, Guliyev E, Reuter C, Budden M, Zöllner J,-P, Holz M, Reum A, Durrani Z, Jones M, Aydogan C, Bicer M, Alaca B, E, Kühnel M, Fröhlich T, Füssl R, and Manske E, Field-emission scanning probe lithography with self-actuating and self-sensing cantilevers for devices with single digit nanometer dimensions, In: Novel Patterning Technologies 2018 (2018), Ed, by Lenk C, Hofmann M, Lenk S, Ivanov T, Ahmad A, Kästner M, Guliyev E, Reuter C, Budden M, Zöllner J,-P, Holz M, Reum A, Durrani Z, Jones M, Aydogan C, Bicer M, Alaca B, E, Kühnel M, Fröhlich T, Füssl R, and Manske E

31. Jäger G, Manske E, Hausotte T, Büchner H-J (2000) Nanomessmaschine zur abbefehlerfreien Koordinatenmessung (Nano Measuring Machine for Zero Abbe Offset Coordinate-measuring). In: tm Technisches Messen Plattform für Methoden, Systeme und Anwendungen der Messtechnik 67.7- 8/2000, 319. https://doi.org/ 10.1524/teme.2000.67.7-8.319

32. Jäger G, Manske E, Hausotte T, Büchner H-J (2009) The metrological basis and operation of nanopositioning and nanomeasuring machine NMM-1 Metrologische Grundlagen und Wirkungsweise der Nanopositionier-und Messmaschine NMM-1. In: tm-Technisches Messen Plattform für Methoden, Systeme und Anwendungen der Messtechnik 76.5, 227-234. https://doi.org/10.1524/teme. 2009.0960

33. Hausotte T (2018) Interferometric measuring systems of nanopositioning and nanomeasuring machines. https://doi.org/10.1117/ 12.2318309

34. Hausotte T (2011) Nanopositionier- und Nanomessmaschinen Geräte für hochpräzise makro- und nanoskalige Oberflächen- und Koordinatenmessungen

35. Manske E, Fröhlich T, Füssl R, Mastylo R, Blumröder U, Köchert P, Birli O, Ortlepp I, Pruss C, Schwesinger F, Meister A (2019) Scale spanning subnanometer metrology up to ten decades. In: Optical measurement systems for industrial inspection XI 11056. Ed. by Lehmann, P., Osten, W., and Jr., A. A. G., pp. 130-136. https://doi.org/10.1117/12.2526076

36. Balzer GF (Nov. 2015) Entwicklung und Untersuchungen zur 3-D-Nanopositioniertechnik in grossen Bewegungsbereichen. $\mathrm{PhD}$ thesis. Technische Universität Ilmenau

37. Friedrich-Alexander-Universität, Lehrstuhl für Fertigungsmesstechnik, Messtechnische Ausstattung (2020). https://www. fmt.tf.fau.de/forschung/messtechnische-ausstattung/ 
38. Osten W (2018) Optical metrology: the long and unstoppable way to become an outstanding measuring tool. In: Speckle 2018: VII international conference on speckle metrology. Ed. by Józwik, M., Jaroszewicz, L. R., and Kujawiska, M. SPIE. https://doi.org/10. $1117 / 12.2322533$

39. Manske E, Fröhlich T, Füssl R, Ortlepp I, Mastylo R, Blumröder U, Dontsov D, Kuehnel M, Koechert P (2020) Progress of nanopositioning and nanomeasuring machines for cross-scale measurement with sub-nanometre precision. Measurement Science and Technology. https://doi.org/10.1088/1361-6501/ab848c

40. Stauffenberg J, Reuter C, Ortlepp I, Holz M, Dontsov D, Schäffel C, Zöllner J-P, Range low I, Strehle S, Manske E (2021) Nanopositioning and fabrication using the nano fabrication machine with a positioning range up to $\varnothing 100 \mathrm{~mm}$. In: Novel patterning technologies 2021. Ed. by Panning, E. M. and Liddle, J. A. Vol. 11610. International Society for Optics and Photonics. SPIE, pp. 82-91. https://doi.org/10.1117/12.2583703

41. Ortlepp I, Kühnel M, Hofmann M, Weidenfeller L, Kirchner J, Supreeti S, Mastylo R, Holz M, Michels T, Füssl R, Rangelow I, Fröhlich T, Dontsov D, Schäffel C, Manske E (2020) Tip- and laser-based nanofabrication up to $100 \mathrm{~mm}$ with sub-nanometre precision. In: Novel Patterning Technologies for Semiconductors, MEMS / NEMS and MOEMS 2020. Ed. by Panning, E. M. and Sanchez, M. I. SPIE. https://doi.org/10.1117/12.2551044

42. Jäger G, Hausotte T, Manske E, Büchner H-J, Mastylo R, Dorozhovets N, Hofmann N (2010) Nanomeasuring and nanopositioning engineering. Measurement 43(9):1099-1105. https:// doi.org/10.1016/j.measurement.2010.04

43. Fischer J, Wegener M (2013) Three-dimensional optical laser lithography beyond the diffraction limit. Laser Photon Rev 7(1):22-44. https://doi.org/10.1002/lpor.201100046

44. Weidenfeller L, Kirchner J, Hofmann M, Kühnel M, Reinhardt C, Rangelow I, Manske E (2019) Laser-microfabrication with accurate positioning and metrological traceability. In: Advanced fabrication technologies for micro/nano optics and photonics XII. Ed. by Freymann, G. von, Schoenfeld, W. V., and Rumpf, R. C. Vol. 10930. International Society for Optics and Photonics. SPIE, pp. 29-36. https://doi.org/10.1117/12.2508248

45. Meister A, Weidenfeller L, Kirchner J, Hofmann M, Mastylo R, Füssl R, Reger J, Manske E (2019) Trans-scale nanofabrication with nanopositioning and nanomeasuring machines. In: IFACPapers OnLine 52.15. 8th IFAC symposium on mechatronic systems mechatronics, 151-156. https://doi.org/10.1016/j.ifacol. 2019.11.666

46. Weidenfeller L (2020) Kombination von zweiphotonen- basiertem direktem Laserschreiben mit grossflächiger und hochpräziser Nanopositionierung. Technische Universität Ilmenau, Dissertation

47. Kirchner J, Weidenfeller L, Gerhardt U, Mastylo R, Kühnel M, Sinzinger S, Manske E (2019) A combined laser scanning and DLW tool for measuring and fabrication tasks with NPMM. In: Advanced fabrication technologies for micro/nano optics and photonics XII. Ed. by Freymann, G. von, Schoenfeld, W. V., and Rumpf, R. C. Vol. 10930. International Society for Optics and Photonics. SPIE, 106-111. https://doi.org/10.1117/12.2508263

48. Kaestner M, Rangelow IW (2011) Scanning proximal probe lithography for sub-10 $\mathrm{nm}$ resolution on calix[4]resorcinarene. J Vacuum Sci Technol B 29(6):06FD02. 10.1116/1.3662092

49. Woszczyna M, Zawierucha P, Paetko P, Zielony M, Gotszalk T, Sarov Y, Ivanov T, Frank A, Zöllner J-P, Rangelow, IW (2010) Micromachined scanning proximal probes with integrated piezoresistive readout and bimetal actuator for high eigenmode operation. J Vacuum Sci Technol B 28(6): C6N12-C6N17. https://doi. org/10.1116/1.3518465

50. Sarov Y, Ivanov T, Frank A, Zöllner J-P, Nikolov N, Rangelow IW (2010) Realization of cantilever arrays for parallel proximity imaging. J Phys Conf Ser 253:012050. https://doi.org/10.1088/ 1742-6596/253/1/012050

51. Sarov Y, Frank A, Ivanov T, Zöllner J-P, Ivanova K, Volland B, Rangelow IW, Brogan A, Wilson R, Zawierucha P, Zielony M, Gotszalk T, Nikolov N, Zier M, Schmidt B, Kostic I (2009) Parallel proximal probe arrays with vertical interconnections. J Vacuum Sci Technol B Microelectron Nanometer Struct Process Meas Phenomena 27(6), 3132-3138. https://doi.org/10.1116/1.3256662

52. Rangelow I, Ivanov T, Hudek T, Fortagne O (2005) Device and method for mask-less AFM microlithography. US2005225011 (A1)

53. Rangelow IW, Ivanov T, Ahmad A, Kästner M, Lenk C, Bozchalooi IS, Xia F, Youcef- Toumi K, Holz M, Reum A (2017) Review Article: active scanning probes: a versatile toolkit for fast imaging and emerging nanofabrication. In: Journal of vacuum science and technology 6. Ed. by Ivanov, T., Ahmad, A., Kästner, M., Lenk, C., Bozchalooi, I. S., Xia, F., Youcef- Toumi, K., Holz, M., and Reum, A

54. Hofmann M, Weidenfeller L, Supreeti S, Mechold S, Holz M, Reuter C, Sinzinger S, Manske E, Rangelow IW (2020) Mix-andmatch lithography and cryogenic etching for NIL template fabrication. Microelectron Eng. https://doi.org/10.1016/j.mee.2020. 111234

55. Kaestner M, Rangelow IW (2020) Scanning probe lithography on calixarene towards single-digit nanometer fabrication. Int $\mathbf{J}$ Extreme Manufact 2:032005

56. Hofmann M (2018) Selective pattern transfer of nano scale features generated by FE-SPL in $10 \mathrm{~nm}$ thick resist layers. Am J Nano Res Appl 6(1):11. https://doi.org/10.11648/j.nano.20180601.12

57. Hofmann M, Mecholdt S, Mohr M, Holz M, Dallorto S, Manske E, Fecht H-J, Rangelow IW (2019) Nanoscale lift-off process using field emission scanning probe lithography. J Vacuum Sci Technol B 37(6):061803. https://doi.org/10.1116/1.5122272

58. Hofmann M, Lenk C, Ivanov T, Rangelow IW, Reum A, Ahmad A, Holz M, Manske E (2018) Field emission from diamond nanotips for scanning probe lithography. J Vacuum Sci Technol B 36(6):06JL02. https://doi.org/10.1116/1.5048193

59. Meijer J, Schenkel T, Rangelow IW (2021) Single dopant atom lithography for the fabrication of quantum computers and low power electronic devices. In: Novel Patterning Technologies 2021. Ed. by Panning, E. M. and Liddle, J. A. Vol. 11610. International Society for Optics and Photonics. SPIE, pp. 92-100. https://doi. org/10.1117/12.2584612

60. Cao X, Fesser P, Fischer D, Hofmann M, Bourgin Y, Sinzinger S (2018) Der Lau-Effekt in der lithographischen Mikro-Nanostrukturierung. In: 119. Tagung der DGaO

61. Cao X (2020) Innovative Beleuchtung für neuartige Abbildungsund Lithographiesysteme. Technische Universität Ilmenau, Dissertation

62. Fischer D, Sinzinger S (2020) Evaluation of quadratic phase hologram calculation algorithms in the Fourier regime. Appl Opt 59(6):1501-1506. https://doi.org/10.1364/AO.381547

63. Supreeti S, Kirchner J, Hofmann M, Mastylo R, Rangelow IW, Manske E, Hoffmann M, Sinzinger S (2019) Integrated soft UVnanoimprint lithography in a nanopositioning and nanomeasuring machine for accurate positioning of stamp to substrate. In: Novel Patterning Technologies for Semiconductors, MEMS/NEMS, and MOEMS 2019. Ed. by Sanchez, M. I. and Panning, E. M. Vol. 10958. International Society for Optics and Photonics. SPIE, pp 151-157. https://doi.org/10.1117/12.2514832

64. Reichle R, Häfner M, Pruss C, Osten W (2010) Diffraktive Strukturen auf gekrümmten Oberflächen für hybride Abbildungssysteme (Diffractive structures on curved surfaces for hybrid imaging systems). Photonik 4:36-40

65. Leuthold J, Hoessbacher C, Muehlbrandt S, Melikyan A, Kohl M, Koos C, Freude W, Dolores-Calzadilla V, Smit M, Suarez I, 
Martínez- Pastor J, Fitrakis E, Tomkos I (2013) Plasmonic communications: light on a wire. Opt Photon News 24(5):28-35. https://doi.org/10.1364/OPN.24.5.000028

66. Cheng Y, Liao Y, Sugioka K (2014) Femtosecond laser 3D nanofabrication in glass: enabling direct write of integrated micro/ nanofluidic chips. In: 8967. Ed. by Nakata, Y., Xu, X., Roth, S., and Neuenschwander, B., pp. 24-35. https://doi.org/10.1117/12. 2042742

67. Fern F (2020) Metrologie in fünfachsigen Nanomess- und Nanopositioniermaschinen. Dissertation. Technische Universität Ilmenau

68. Schienbein R (2020) Grundlegende Untersuchungen zum konstruktiven Aufbau von Fünfachs-Nanopositionier- und Nanomessmaschinen. Dissertation. Technische Universität Ilmenau

69. Ortlepp I, Fern F, Schienbein R, Supreeti S, Füssl R, Theska R, Sinzinger S, Manske E (2021) Traceable 5D-Nanofabrication with Nano Positioning and Nano Measuring Machines. In: euspens 21st international conference \& exhibition, Copenhagen, DK

70. Weise C, Wulff K, Reger J (2018) Observer with improved convergence for a class of initialized FO-LTI systems. In: 2018 IEEE conference on control technology and applications (CCTA). pp 1570-1576. https://doi.org/10.1109/CCTA.2018.8511326

71. Weise C, Wulff K, Reger J (2019) Fractional-order memory reset control for integer-order LTI systems. In: 2019 IEEE 58th conference on decision and control (CDC). pp 5710- 5715. https://doi. org/10.1109/CDC40024.2019.9029825

72. Weise C, Wulff K, Reger J (2017) Fractional-order observer for integer-order LTI systems. In: pp 2101-2106. https://doi.org/10. 1109/ASCC.2017.8287499

73. Devasia S, Eleftheriou E, Moheimani SOR (2007) A survey of control issues in nanopositioning. IEEE Trans Control Syst Technol 15(5):802-823. https://doi.org/10.1109/TCST.2007.903345
74. Sunny MR, Kapania RK, Moffitt RD, Mishra A, Goulbourne N (2010) A modified fractional calculus approach to model hysteresis. J Appl Mech. https://doi.org/10.1115/1.4000413

75. Gorges S, Hesse, S (2018) Design and modeling approach for a lifting and actuating unit for the application in nano-precision machines. In: Annual Meeting

76. Gorges S (2020) A lifting and actuating unit for a planar nanoprecision drive system. Technische Universität Ilmenau, Dissertation

77. Gorges S, Hesse S, Schäffel C, Ortlepp I, Manske E, Langlotz E, Dontsov D (2019) Integrated planar 6-DOF nanopositioning system. In: IFAC-PapersOnLine 52.15. 8th IFAC symposium on mechatronic systems mechatronics 2019, pp 313-318. https://doi. org/10.1016/j.ifacol.2019.11.693

78. Yan N, Vasilyan S, Fröhlich T (Apr. 2019) Torsion balance-based system for high-precision force measurement in horizontal plane: part II. Static and dynamic improvement. Meas Sci Technol 30(5): 055104. https://doi.org/10.1088/1361-6501/ab05aa

79. Dannberg 0, Kühnel M, Fröhlich T (2020) Entwicklung einer Cantileverkalibriereinrichtung. In: tmTechnisches Messen 87.10, pp 622-629. https://doi.org/10.1515/teme-2020-0064

80. Marangoni RR, Schleichert J, Rahneberg I, Mastylo R, Manske E, Fröhlich T (2017) Multicomponent force measurement in micromachining. In: tmTechnisches Messen 84.9, pp 587-592. https://doi.org/10.1515/teme-2016-0066

81. Seo J-H, Park JH, Kim S-I, Park BJ, Ma Z, Choi J, Ju B-K (2014) Nanopatterning by laser interference lithography: applications to optical devices. J Nanosci Nanotechnol 14(2):1521-1532. https:// doi.org/10.1166/jnn.2014.9199

82. Tong QC, Nguyen DTT, Do MT, Luong MH, Journet B, LedouxRak I, Lai ND (2016) Direct laser writing of polymeric nanostructures via optically induced local thermal effect. Appl Phys Lett 108(8):183104. https://doi.org/10.1063/1.4948589

\section{Authors and Affiliations}

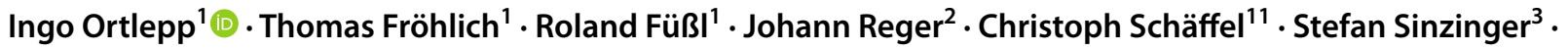
Steffen Strehle ${ }^{4} \cdot$ René Theska $^{5}$. Lena Zentner ${ }^{6}$. Jens-Peter Zöllner ${ }^{7} \cdot$ Ivo W. Rangelow ${ }^{8}$. Carsten Reinhardt ${ }^{12}$. Tino Hausotte ${ }^{13}$. Xinrui Cao ${ }^{3}$. Oliver Dannberg ${ }^{1} \cdot$ Florian Fern $^{14}$. David Fischer ${ }^{3}$. Stephan Gorges ${ }^{11}$. Martin Hofmann ${ }^{4}$. Johannes Kirchner ${ }^{15}$. Andreas Meister ${ }^{9} \cdot$ Taras Sasiuk $^{1} \cdot$ Ralf Schienbein $^{5}$. Shraddha Supreeti ${ }^{10}$. Laura Mohr-Weidenfeller ${ }^{4} \cdot$ Christoph Weise $^{2} \cdot$ Christoph Reuter $^{4} \cdot$ Jaqueline Stauffenberg $^{1} \cdot$ Eberhard Manske $^{1}$

1 Institute of Process Measurement and Sensor Technology, Technische Universität Ilmenau, Ehrenbergstrasse 29, 98693 Ilmenau, Germany

2 Control Engineering Group, Technische Universität Ilmenau, Ilmenau, Germany

3 Optical Engineering Group, Technische Universität Ilmenau, Ilmenau, Germany

4 Microsystems Technology Group, Technische Universität Ilmenau, Ilmenau, Germany

5 Precision Engineering Group, Technische Universität Ilmenau, Ilmenau, Germany

6 Compliant Systems Group, Technische Universität Ilmenau, Ilmenau, Germany

7 Micro- and Nanoelectronic Systems Group, Technische Universität Ilmenau, Ilmenau, Germany

8 Nanoscale Systems Group, Technische Universität Ilmenau, Ilmenau, Germany
9 Division for Research Service and Technology Transfer, Technische Universität Ilmenau, Ilmenau, Germany

10 Electronics Technology Group, Technische Universität Ilmenau, Ilmenau, Germany

11 IMMS Institut für Mikroelektronik- und Mechatronik-Systeme Gemeinnützige GmbH (IMMS $\mathrm{GmbH}$ ), Ilmenau, Germany

12 Fachgebiet Technische Physik, City University of Applied Sciences, Bremen, Germany

13 Lehrstuhl für Fertigungsmesstechnik, Friedrich-Alexander-U niversität (FAU), Erlangen-Nürnberg, Germany

14 JUMO GmbH \& Co. KG, Fulda, Germany

15 Asphericon GmbH, Jena, Germany 